\title{
Kerr nonlinear coupler and entanglement
}

\author{
Wiesław Leoński ${ }^{1}$ and Adam Miranowicz ${ }^{1,2}$ \\ ${ }^{1}$ Institute of Physics, Adam Mickiewicz University, ul. Umultowska 85, 61-614 Poznań, Poland \\ ${ }^{2}$ CREST Research Team for Interacting Carrier Electronics, \\ Graduate University for Advanced Studies (SOKENDAI), Hayama, Kanagawa 240-0193, Japan
}

\begin{abstract}
We discuss a model comprising two coupled nonlinear oscillators (Kerr-like nonlinear coupler) with one of them pumped by an external coherent excitation. Applying the method of nonlinear quantum scissors we show that the quantum evolution of the coupler can be closed within a finite set of $n$-photon Fock states. Moreover, we show that the system is able to generate Bell-like states and, as a consequence, the coupler discussed behaves as a two-qubit system. We also analyze the effects of dissipation on entanglement of formation parametrized by concurrence.
\end{abstract}

PACS numbers: 42.50Dv, 03.67Mn

\section{INTRODUCTION}

Quantum entanglement seems to be one of the most striking phenomena of quantum physics. It is not only one of the most fundamental concepts of quantum information theory, but also allows investigation of many features of nonlocal properties of quantum systems [1]. Various aspects of the entanglement and its generation have been discussed in numerous papers, especially from the point of view of quantum information applications including quantum key distribution [2], superdense coding [3], quantum teleportation [4], fast quantum computations [5, 6], entanglement-assisted communication [] or broadcasting of entanglement [8].

In this paper we shall concentrate on the dynamics of the Kerr nonlinear coupler and its ability to produce quantum entangled states. Since the pioneering works of Jensen 9] and Maier [10, the nonlinear couplers attract an increasing interest 11]-17] (for reviews see [18, 19]). As shown in classical [9, 10] and quantum [1] models, the Kerr couplers can exhibit self-trapping, self-modulation and self-switching of the energy of the coupled modes. These phenomena have potential applications in optical communications as, e.g., intensity-dependent routing switches. Among various other quantum statistical properties, it has been shown that the Kerr couplers can be a source of sub-Poissonian and squeezed light [12]- 16]. Another group of papers concerns the correspondences between the quantum and classical dynamics of such systems [1] and their chaotic dynamics, including synchronization effects [17].

Quantum optical systems based on Kerr nonlinearity have been applied for various quantum information purposes including entanglement purification [20], complete quantum teleportation 21], or realization of qubit phase gates 22]. Here, we present another simple quantum information application Kerr nonlinearities, namely for generation of entangled optical qubits from classical light.

We are interested here in a simple model comprising two quantum nonlinear oscillators located inside one cavity. These oscillators are linearly coupled to each other, while one of the oscillators is excited by an external co-

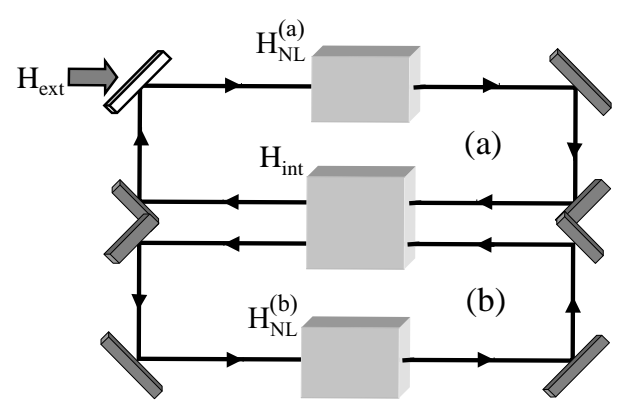

FIG. 1: Scheme of a pumped nonlinear coupler, described by Hamiltonian (1), implemented by two ring cavities ( $a$ and $b$ ) filled with Kerr media, where cavity $a$ is being excited by a single-mode external classical field.

herent field of a constant amplitude. For this model we shall answer the questions, whether it is possible to close the dynamics of the excited nonlinear coupler within a finite set of $n$-photon states and, which is the main subject of this paper, whether nonlinear excited coupler can be a source of maximally entangled (ME) states.

\section{THE MODEL AND SOLUTIONS}

The model of the Kerr nonlinear coupler discussed here contains two nonlinear oscillators linearly coupled to each other and, additionally, one of them is coupled to an external coherent field as presented in figure 1. We assume, that this excitation is linear and has a constant amplitude. This system can be described by the following Hamiltonian:

$$
\hat{H}=\hat{H}_{N L}+\hat{H}_{i n t}+\hat{H}_{e x t}
$$

where

$$
\begin{aligned}
\hat{H}_{N L} & \equiv \hat{H}_{N L}^{(a)}+\hat{H}_{N L}^{(b)}=\frac{\chi_{a}}{2}\left(\hat{a}^{\dagger}\right)^{2} \hat{a}^{2}+\frac{\chi_{b}}{2}\left(\hat{b}^{\dagger}\right)^{2} \hat{b}^{2} \\
\hat{H}_{\text {int }} & =\epsilon \hat{a}^{\dagger} \hat{b}+\epsilon^{*} \hat{a} \hat{b}^{\dagger} \\
\hat{H}_{\text {ext }} & =\alpha \hat{a}^{\dagger}+\alpha^{*} \hat{a}
\end{aligned}
$$


We see that $\hat{H}_{N L}$ describes nonlinear oscillators, $\hat{H}_{\text {int }}$ corresponds to an internal coupling, whereas the term $\hat{H}_{\text {ext }}$ describes a linear coupling between the external field and the mode of the field inside our cavity corresponding to the oscillator $a$. The parameters $\chi_{a}$ and $\chi_{b}$ are nonlinearity constants of the oscillators $a$ and $b$, respectively, $\epsilon$ describes the strength of the oscillator oscillator coupling, whereas $\alpha$ is a strength of the external excitation of the oscillator $a$. It is worth noting that our Hamiltonian $\hat{H}_{\text {int }}$ does not include nonlinear coupling proportional to $\hat{b}^{\dagger} \hat{b} \hat{a}^{\dagger} \hat{a}$ but only the linear one, described by (3). Nevertheless, the same Hamiltonian $\hat{H}_{N L}+\hat{H}_{\text {int }}$ as ours was used, e.g., by Bernstein [23] and Chefles and Barnett [1] to describe the nonlinear coupler.

In the first part of our analysis we neglect damping processes in our model, thus the system evolution can be described by a time-dependent wave-function. This function can be written in the $n$-photon Fock basis as:

$$
|\psi(t)\rangle=\sum_{n, m=0}^{\infty} c_{n, m}(t)|n\rangle_{a}|m\rangle_{b}
$$

where $c_{n, m}(t)$ is a complex probability amplitude of finding our system in the $n$-photon and $m$-photon states for the mode $a$ and $b$, respectively.

We have included here an external coupling and therefore, the energy inside the cavity is not conserved. As a consequence, we can expect that in the evolution of the system many of the states corresponding to great number of photons will be involved. However, we can overcome this difficulty by applying the nonlinear quantum scissors method discussed in [24] (for the discussion concerning quantum states defined in finite-dimensional Hilbert spaces and the methods of their generation see the review papers [25, 26] and the references cited therein). Namely, it is seen from the form of $\hat{H}_{N L}$ that this Hamiltonian produces degenerate levels of the energy equal to zero, corresponding to the following four states: $|0\rangle_{a}|0\rangle_{b}$, $|1\rangle_{a}|0\rangle_{b},|0\rangle_{a}|1\rangle_{b}$ and $|1\rangle_{a}|1\rangle_{b}$. Moreover, all couplings discussed here have constant envelopes, and similarly as in [24], we assume that they are weak. Therefore, we can treat transitions within the mentioned set of the states as that of resonant nature. The evolution of discussed system is closed within the set of these four states and interactions with other states can be neglected in our approximation. Thus, the wave-function describing our model can be written in the form:

$$
\begin{aligned}
|\psi(t)\rangle= & c_{0,0}(t)|0\rangle_{a}|0\rangle_{b}+c_{1,0}(t)|1\rangle_{a}|0\rangle_{b} \\
& +c_{0,1}(t)|0\rangle_{a}|1\rangle_{b}+c_{1,1}(t)|1\rangle_{a}|1\rangle_{b}
\end{aligned}
$$

and hence, the equations of motion for the system are:

$$
\begin{aligned}
i \frac{d}{d t} c_{0,0} & =\alpha^{*} c_{1,0}, \\
i \frac{d}{d t} c_{1,0} & =\epsilon c_{0,1}+\alpha c_{0,0}, \\
i \frac{d}{d t} c_{0,1} & =\epsilon^{*} c_{1,0}+\alpha^{*} c_{1,1},
\end{aligned}
$$

$$
i \frac{d}{d t} c_{1,1}=\alpha c_{0,1} \text {. }
$$

To solve these equations we need to find roots of the forth-order polynomial, which leads to a very complicated and unreadable form of final formulas. Therefore, although it is formally possible, we shall not write the analytical solutions in their most general form and we will restrict our considerations to the case of real $\alpha=\epsilon$. Moreover, we assume that for the time $t=0$ the both oscillators were in vacuum states, i.e.,

$$
|\psi(t=0)\rangle=|0\rangle_{a}|0\rangle_{b} .
$$

Then we get the following solutions for the probability amplitudes $c_{i, j}(i, j=0,1)$ :

$$
\begin{aligned}
& c_{0,0}(t)=\cos (x t) \cos (y t)+\frac{1}{\sqrt{5}} \sin (x t) \sin (y t), \\
& c_{1,0}(t)=-i \frac{2}{\sqrt{5}} \cos (x t) \sin (y t), \\
& c_{0,1}(t)=-\frac{2}{\sqrt{5}} \sin (x t) \sin (y t), \\
& c_{1,1}(t)=i\left[\frac{1}{\sqrt{5}} \cos (x t) \sin (y t)-\sin (x t) \cos (y t)\right]
\end{aligned}
$$

where $x=\alpha / 2$ and $y=\sqrt{5} x$. The solution (9) is valid under the condition $\chi_{j} \gg \epsilon=\alpha(j=a, b)$, which implies that it is apparently independent of nonlinearities $\chi_{j}$. But it should be stressed that the corresponding nonlinear Hamiltonian $\hat{H}_{N L}$, given by (2), is responsible for the truncation of the infinite-dimensional state to the finite superposition, given by (6). Otherwise, if $\chi_{j}$ were not much stronger than $\epsilon$ and $\alpha$, the state generated would not be truncated to the finite superposition (66) and the probability amplitudes $c_{n, m}(t)$ would depend explicitly on nonlinearities $\chi_{j}$.

To check our solutions we can calculate the probability amplitudes numerically in a basis expanded to the states corresponding to greater number of photons than discussed here (for the model discussed our considerations are restricted by the resonances to the one-photon and vacuum states only). We perform the calculations following the method discussed in [26], and first we construct the unitary evolution operator $\hat{U}$ applying the full Hamiltonian shown in (1):

$$
\hat{U}=\exp (-i \hat{H} t) .
$$

Then we are able to obtain the wave-function $|\psi(t)\rangle$ by acting the operator $\hat{U}$ on the initial state of the system, and for the case discussed here we have:

$$
|\psi(t)\rangle=\hat{U}(t)|0\rangle_{a}|0\rangle_{b} .
$$

Figure 2 shows both analytical and numerical results of our calculations. We see very good agreement between these two methods, so the model based on the resonances 


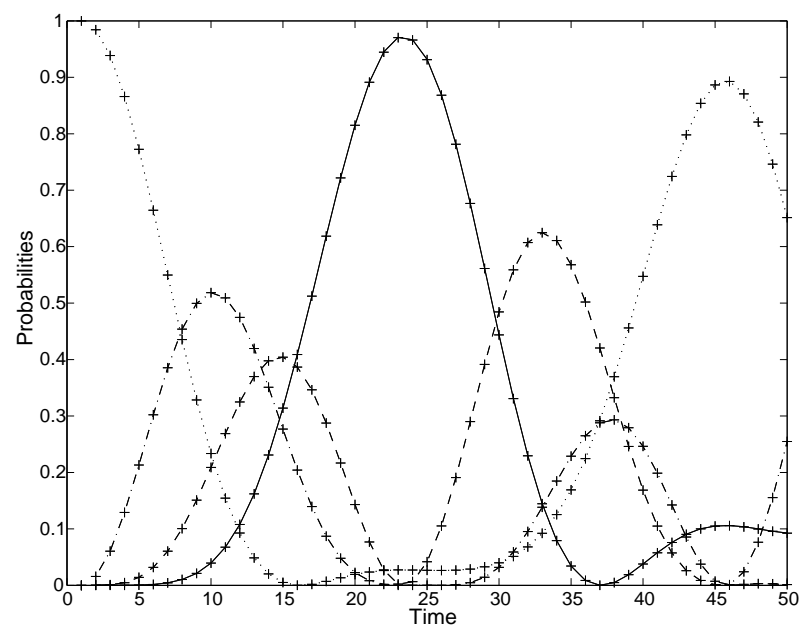

FIG. 2: Probabilities for finding the coupler in the $|0\rangle_{a}|0\rangle_{b}$ (dotted curve), $|1\rangle_{a}|0\rangle_{b}$ (dashed-dotted curve) $|0\rangle_{a}|1\rangle_{b}$ (dashed curve) and $|1\rangle_{a}|1\rangle_{b}$ (solid curve) states from the analytical results and their numerical counterparts (cross marks). The nonlinearity coefficients $\chi_{a}=\chi_{b}=25$ and the coupling strengths $\epsilon=\alpha=\pi / 25$.

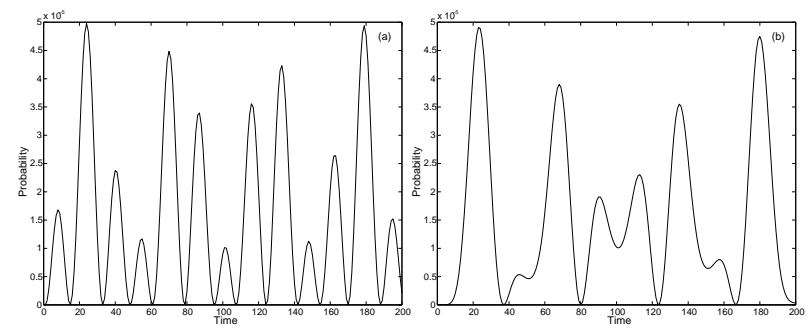

FIG. 3: The same as in figure 2 but for the states: (a) $|0\rangle_{a}|2\rangle_{b}$ and (b) $|1\rangle_{a}|2\rangle_{b}$.

works very well. Moreover, from the numerical results for the probabilities corresponding to the states $|0\rangle_{a}|2\rangle_{b}$ (figure 3a) and $|1\rangle_{a}|2\rangle_{b}$ (figure 3b), we see that the states corresponding to the numbers of photons higher than one are practically unpopulated. It is worth mentioning that our numerical calculations have been performed in the $m$ dimensional Fock basis, where $m \simeq 20$ for each subspace associated with a single mode of the field.

\section{COUPLER AND ENTANGLEMENT}

The time-evolution of the probability amplitudes can give some information concerning entanglement in our system too. For instance, if we see in a figure that probability corresponding to one of the discussed states is equal to another one and additionally, the both are equal to $1 / 2\left(\left|c_{i, j}\right|^{2}=\left|c_{k, l}\right|^{2}=0.5\right.$ for every $\left.i, j, k, l\right)$, we know that the system generates ME states. Obviously, this method of finding entangled states is not very accurate, especially for the case when we should observe and compare various and often rapidly oscillating probabilities. Therefore, we apply another method convenient for find-
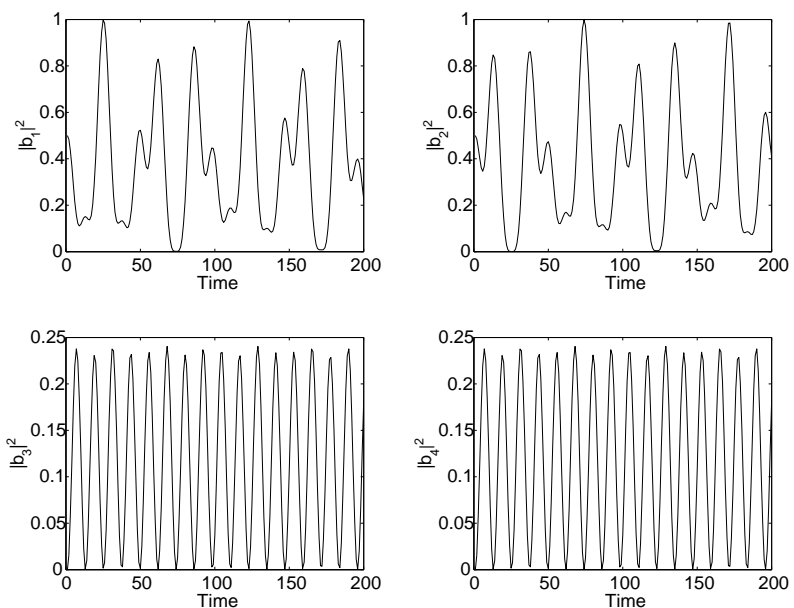

FIG. 4: Probabilities for finding the coupler in the Belllike states. The nonlinearity coefficients $\chi_{a}$ and $\chi_{b}$, and the coupling strengths $\epsilon$ and $\alpha$ are identical to those of figures $2-3$.

ing entanglement in the system. Namely we shall express obtained wave-function in the Bell basis:

$$
|\psi\rangle=b_{1}\left|B_{1}\right\rangle+b_{2}\left|B_{2}\right\rangle+b_{3}\left|B_{3}\right\rangle+b_{4}\left|B_{4}\right\rangle
$$

where the states $\left|B_{i}\right\rangle, i=1,2,3,4$ are Bell-like states that can be expressed as functions of discussed here $n$ photon states (Bell-like states differ from the commonly discussed Bell states in the existence of the phase factor - for the case discussed here, one of the $n$-photon states is multiplied by $i)$ :

$$
\begin{aligned}
\left|B_{1}\right\rangle & =\frac{1}{\sqrt{2}}\left(|1\rangle_{a}|1\rangle_{b}+i|0\rangle_{a}|0\rangle_{b}\right), \\
\left|B_{2}\right\rangle & =\frac{1}{\sqrt{2}}\left(|0\rangle_{a}|0\rangle_{b}+i|1\rangle_{a}|1\rangle_{b}\right), \\
\left|B_{3}\right\rangle & =\frac{1}{\sqrt{2}}\left(|0\rangle_{a}|1\rangle_{b}-i|1\rangle_{a}|0\rangle_{b}\right), \\
\left|B_{4}\right\rangle & =\frac{1}{\sqrt{2}}\left(|1\rangle_{a}|0\rangle_{b}-i|0\rangle_{a}|1\rangle_{b}\right) .
\end{aligned}
$$

These states are maximally entangled states, and therefore, for the cases when $\left|b_{i}\right|^{2}=1, i=1,2,3,4$ also our system evolves into ME state. Figure 4 shows probabilities corresponding to the Bell-like states as a function of time. Moreover, all parameters describing our system are identical to those of figure 2 . We see, that for the time $t \simeq 115$ we get the state $\left|B_{1}\right\rangle$ and for $t \simeq 80$ the state $\left|B_{2}\right\rangle$ is generated with high accuracy - our system becomes maximally entangled. This entanglement involves the states $|0\rangle_{a}\left|0_{b}\right\rangle$ and $|1\rangle_{a}\left|1_{b}\right\rangle$. Of course, one should keep in mind that plots in figure 4 are for the probabilities not for their complex amplitudes and hence, we get the Bell-like states from (13) with some phase factor. Nevertheless, our states are maximally entangled. Moreover, figure 4 shows that the values of probabilities for the states $\left|B_{3}\right\rangle$ and $\left|B_{4}\right\rangle$ can reach maximally 0.8 . 
As a consequence, the states $|1\rangle_{a}\left|0_{b}\right\rangle$ and $|0\rangle_{a}\left|1_{b}\right\rangle$ cannot be maximally entangled for the initial vacuum states $|0\rangle_{a}\left|0_{b}\right\rangle$. But generation of $\left|B_{3}\right\rangle$ and $\left|B_{4}\right\rangle$ would be possible by assuming that the system is initially in the states $|1\rangle_{a}\left|0_{b}\right\rangle$ or $|0\rangle_{a}\left|1_{b}\right\rangle$.

The Bell-like states (13) are maximally entangles, however, they are not the only entangled states that could be produced by the system. Therefore, to measure the entanglement degree of the system we apply the measure that is referred to as the concurrence. This quantity proposed by Wootters [27] is one of the most commonly applied measures of the entanglement. The concurrence for two qubit states is defined as

$$
\mathcal{C}=\max \left\{0, \lambda_{1}-\lambda_{2}-\lambda_{3}-\lambda_{4}\right\}
$$

where $\lambda_{i}(i=1, \ldots, 4)$ are the square roots of the eigenvalues of the matrix

$$
\tilde{\rho}=\rho\left(\sigma_{y}^{a} \otimes \sigma_{y}^{b}\right) \rho^{*}\left(\sigma_{y}^{a} \otimes \sigma_{y}^{b}\right)
$$

where $\sigma_{y}^{\{a, b\}}$ are Pauli matrices defined in subspaces corresponding to the modes $\{a, b\}$, and the eigenvalues $\lambda_{i}$ appearing in (14) should be taken in decreasing order. Concurrence takes values from 0 to 1 , where for unentangled states it vanishes, whereas for ME states it is equal to one.

Damping is the main and unavoidable source of decoherence which can easily destroy entangled states. Hence, for our results to be applicable in real physical systems, we present a numerical analysis of the damping effects on the concurrence. Let us assume that the leakage of photons from the cavities $a$ and $b$ is described by the rates $\kappa_{a}$ and $\kappa_{b}$, respectively. Starting from Hamiltonian (11) and defining the collapse operators by $\hat{C}_{a}=\sqrt{2 \kappa_{a}} \hat{a}$ and $\hat{C}_{b}=\sqrt{2 \kappa_{b}} \hat{b}$, we can write the time-independent Liouvillian in the standard Linblad form

$$
\hat{\mathcal{L}} \hat{\rho}=-i[\hat{H}, \hat{\rho}]+\sum_{j=a, b}\left(\hat{C}_{j} \hat{\rho} \hat{C}_{j}^{\dagger}-\frac{1}{2}\left(\hat{C}_{j}^{\dagger} \hat{C}_{j} \hat{\rho}+\hat{\rho} \hat{C}_{j}^{\dagger} \hat{C}_{j}\right)\right)
$$

The evolution of the density matrix $\hat{\rho}(t)$ in the dissipative system can be found numerically as a series of complex exponentials $\exp \left(\sigma_{k} t\right)$ given in terms of the eigenvalues $\sigma_{k}$ of the Liouvillian $\hat{\mathcal{L}}$, given by (16).

Figure 5 shows the plot of the concurrence evolution for the case discussed here - we have single external excitation of the coupler and we assume that all couplings existing in the system are weak $(\alpha=\epsilon=\pi / 25)$ in comparison to Kerr nonlinearities $\chi_{a}=\chi_{b}=25$. Various curves in figure 5 correspond to concurrence evolutions with different dissipation rates. We see that the varying in time concurrence is modulated by an oscillation of low frequency. As a consequence, several maxima appearing here are of various values. Two of them for dissipationfree evolution (depicted by solid curve), which are the closest to unity, correspond to the formation of Bell-like states $\left|B_{1}\right\rangle$ and $\left|B_{2}\right\rangle$ discussed earlier and shown in figure 4. As a consequence, we can treat our system as a

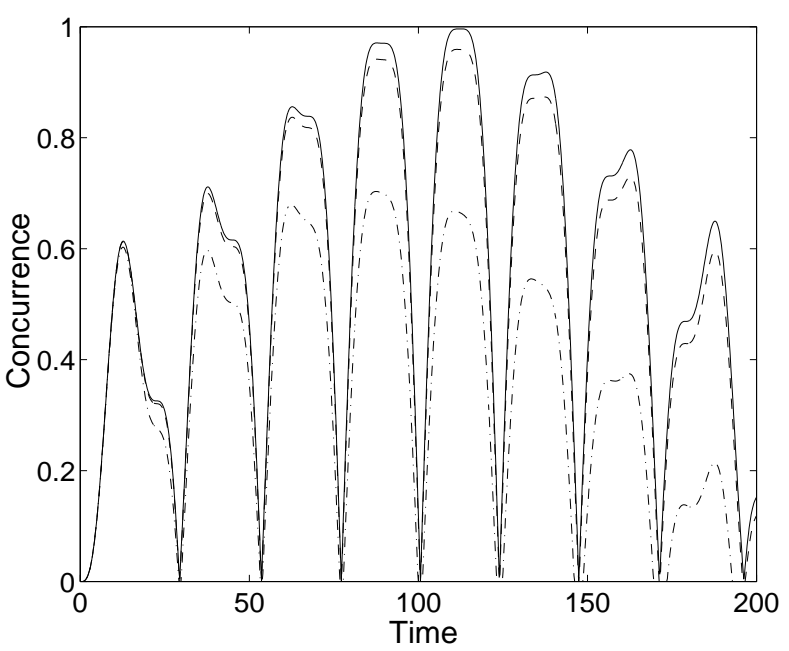

FIG. 5: Concurrence for the excited nonlinear coupler for various cavity leakage rates $\kappa_{a}=\kappa_{b}$ equal to 0 (solid curve), $10^{-4}$ (dashed curve) and $10^{-3}$ (dot-dashed curve). The quantities $\chi_{a}, \chi_{b}, \epsilon$ and $\alpha$ are identical to those of figure 4 .

source of ME states for low dissipation. On the scale of figure 5 and for the chosen coupling parameters, the differences between the evolution with the leakage rates $\kappa_{a}=\kappa_{b} \leq 10^{-5}$ and the dissipation-free evolution are invisible. However, higher leakage rates beyond short time evolution cause essential deterioration of the concurrence limiting the effective generation of ME states. Thus, the results of our calculations indicate that the system discussed is highly sensitive to the dissipation processes. Even relatively small losses from the cavity are able to destroy the entanglement. Therefore, we should assume that we deal here with a very high $Q$ cavity that is capable to preserve practically the whole radiation field located inside. However, this assumption is very desirable from our point of view. For this case the coupler can be weakly excited by external fields only - the less photons can escape from the cavity through the mirror, the smaller number of photons can be injected inside this way.

\section{CONCLUSIONS}

In this paper we have discussed a model of nonlinear coupler linearly excited by a single-mode coherent field. We have shown that the evolution of the system is closed within a finite set of states and only $|i\rangle_{a}|j\rangle_{b}(i, j=0,1)$ states are populated. We have applied here the method used for the nonlinear quantum scissors 24] and have found some analytical formulas for the probability amplitudes corresponding to these states. We have shown that starting from the vacuum state $|0\rangle_{a}|0\rangle_{b}$ of our system, its evolution leads to Bell-like states generation. Moreover, we have calculated the concurrence and its behaviour indicates that the ME states are produced for the system 
if the photon leakage rates out of the cavities are less than $10^{-5}$ for the chosen coupling and nonlinearity parameters. Moreover, we have shown that the concurrence exhibits some modulation effect as a result of the existence of various couplings in our system. For each of the couplings we have some frequency and their interference leads to some long-frequency oscillations in the system.

We see, that our model, despite its simplicity, exhibits intriguing features. We can say that the properties of the system discussed here are much desired from the point of view of the physical properties of the nonlinear couplers. Our scheme can be used for generation of the entangled optical qubits from classical light, which is a basic but rather simple quantum information problem. Introduction of conditional measurement in this scheme, along the lines of Duan et al. proposal 20], is probably worth of further study from the point of view of more sophisticated quantum information applications 20, 21, 22].

Finally, we mention about the experimental feasibility of the presented scheme. Since our solution is applicable only when the cavity-field intensities are very small, so an objection arises that the Kerr nonlinearities are usually negligible in this case. However, the recent breakthrough advances on nonlinear optics involving very weak light fields show that the nonlinearities can be enhanced by several orders of magnitude in ultracold atomic systems using electromagnetically induced transparency when resonant optical absorption is eliminated (28) and references therein). In particular, giant Kerr nonlinearities have been theoretically predicted 29] and first experimentally measured to be $\sim 10^{6}$ greater than those in the conventional optical materials [30]. Thus, we believe that the scheme discussed here can be feasible experimentally.

\section{Acknowledgments}

Authors wish to thank Profs. Ryszard Tanaś, Jarosław Zaremba and Jiří Bajer for their valuable discussions and suggestions.
[1] Einstein A, Podolski B, and Rosen N 1935 Phys. Rev. 47 777

[2] Ekert A K 1991 Phys. Rev. Lett. 67661

Bennett C H, Brassard G, and Mermin N D 1992 Phys. Rev. Lett. 68557

[3] Bennett C H and Wiesner S J 1992 Phys. Rev. Lett. 69 2881

[4] Bennett Ch H, Brassard G, Crépeau C, Jozsa R, Peres A, and Wootters W K 1993 Phys. Rev. Lett. 701895

[5] Shor P W 1997 SIAM J. Comp. 261484 and references therein

[6] Grover L K 1997 Phys. Rev. Lett. 79325

[7] Bennett C H, Fuchs C A and Smolin J A 1997 in: Quantum Communication, Computing, and Measurement, eds O Hirota et al. (New York: Plenum Press) p 79

[8] Bužek V, Vedral V, Plenio M B, Knight P L, and Hillery M 1997 Phys. Rev. A 553327

[9] Jensen S M 1982 IEEE J. Quantum Elect. QE-18 1580

[10] Maier A M 1982 Kvant. Elektron. (Moscow) 92996

[11] Chefles A and Barnett S M 1996 J. Mod. Opt. 43709

[12] Peřina J and Bajer J 1995 J. Mod. Opt. 422337

[13] Korolkova N and Peřina J 1997 Opt. Commun. 136 135; 1997 J. Mod. Opt. 41525

[14] Fiurášek J, Křepelka J, and Peřina J 1999 Opt. Commun. 167115

[15] Ibrahim A -B M A, Umarov B A, Wahiddin M R B 2000 Phys. Rev. A 61043804

[16] Ariunbold G and Perina J (2000) Opt. Commun. 176
149; (2001) J. Mod. Opt. 481005

[17] Grygiel K and Szlachetka P 2001 J. Opt. B: Quant. Semiclass. Opt. 3104

[18] Peřina J Jr and Peřina J 2000 Progress in Optics, ed. E Wolf (Amsterdam: Elsevier) 41361

[19] Fiurášek J and Peřina J 2001 in Coherence and Statistics of Photons and Atoms, ed J Peřina (New York: Wiley) 65

[20] Duan L -M, Giedke G, Cirac J I, and Zoller P 2000 Phys. Rev. Lett. 844002

[21] Vitali D, Fortunato M, and Tombesi P 2000 Phys. Rev. Lett. 85445

[22] Ottaviani C, Vitali D, Artoni M, Cataliotti F, and Tombesi P 2003 Phys. Rev. Lett. 90197902

[23] Bernstein L J 1993 Physica D 68174

[24] Leoński W 1997 Phys. Rev. A. 553874

[25] Miranowicz A, Leoński W, and Imoto N 2001 Adv. Chem. Phys. (New York: Wiley) 119 (I) 155

[26] Leoński W and Miranowicz A 2001 Adv. Chem. Phys. (New York: Wiley) 119 (I) 195

[27] Wootters W K 1998 Phys. Rev. Lett 802245

[28] Lukin M D and Imamoğlu A 2001 Nature (London) 413 273

[29] Schmidt H and Imamoğlu A 1996 Opt. Lett. 211936

[30] Hau L V, Harris S E, Dutton Z, and Behroozi C H 1999 Nature (London) 397594 